\title{
Applications of the Inverse Theta Number in Stable Set Problems
}

\author{
Miklós Ujvári*
}

\begin{abstract}
In the paper we introduce a semidefinite upper bound on the square of the stability number of a graph, the inverse theta number, which is proved to be multiplicative with respect to the strong graph product, hence to be an upper bound for the square of the Shannon capacity of the graph. We also describe a heuristic algorithm for the stable set problem based on semidefinite programming, Cholesky factorization, and eigenvector computation.
\end{abstract}

Keywords: Shannon capacity, stability number, inverse theta number

\section{Introduction}

An algorithm for the stable set problem is useful in many ways, e.g. it can be used for colouring a graph: find a stable set, remove it from the graph, and iterate the algorithm. (See [2] for further applications and approximation algorithms for the stable set problem.) The strength of the semidefinite programming approach for the stable set and colouring problems is shown by the algorithms of GrötschelLovász-Schrijver, Karger-Motwani-Sudan, and Alon-Kahale, see [5] for a summary of these results. In this paper we will describe a heuristic algorithm for the stable set problem based on semidefinite optimization, and the notion of the inverse theta number.

We start the paper with stating the main results. First we fix some notation.

Let $n \in \mathcal{N}$, and let $G=(V(G), E(G))$ be an undirected graph, with vertex set $V(G)=\{1, \ldots, n\}$, and with edge set $E(G) \subseteq\{\{i, j\}: i \neq j\}$. Let $A(G)$ be the $0-1$ adjacency matrix of the graph $G$, that is let

$$
A(G):=\left(a_{i j}\right) \in\{0,1\}^{n \times n}, \text { where } a_{i j}:= \begin{cases}0, & \text { if }\{i, j\} \notin E(G), \\ 1, & \text { if }\{i, j\} \in E(G) .\end{cases}
$$

The complementary graph $\bar{G}$ is the graph with adjacency matrix

$$
A(\bar{G}):=J-I-A(G),
$$

*H-2600 Vác, Szent János utca 1., Hungary. E-mail: ujvarim@cs.elte.hu 
where $I$ is the identity matrix, and $J$ denotes the matrix with all elements equal to one. The disjoint union of the graphs $G_{1}$ and $G_{2}$ is the graph $G_{1}+G_{2}$ with adjacency matrix

$$
A\left(G_{1}+G_{2}\right):=\left(\begin{array}{cc}
A\left(G_{1}\right) & 0 \\
0 & A\left(G_{2}\right)
\end{array}\right) .
$$

We will use the notation $K_{n}$ for the clique graph, and $K_{s_{1}, \ldots, s_{k}}$ for the complete multipartite graph $\overline{K_{s_{1}}+\ldots+K_{s_{k}}}$. Also, we will denote by $C_{n}$ the $n$-cycle, the polygon graph with $n$ vertices.

Let $\left(\delta_{1}, \ldots, \delta_{n}\right)$ be the sum of the row vectors of the adjacency matrix $A(G)$. The elements of this vector are the degrees of the vertices of the graph $G$. Let $\delta_{G}, \Delta_{G}, \mu_{G}$ be the minimum, maximum, resp. the arithmetic mean of the degrees in the graph.

By Rayleigh's theorem (see [9]) for a symmetric matrix $M=M^{T} \in \mathcal{R}^{n \times n}$ the minimum and maximum eigenvalue, $\lambda_{M}$, resp. $\Lambda_{M}$ can be expressed as

$$
\lambda_{M}=\min _{\|u\|=1} u^{T} M u, \Lambda_{M}=\max _{\|u\|=1} u^{T} M u .
$$

Attainment occurs if and only if $u \in \mathcal{R}^{n}$ is a unit eigenvector corresponding to $\lambda_{M}$ and $\Lambda_{M}$, respectively. The minimum and maximum eigenvalue of the adjacency matrix $A(G)$ will be denoted by $\lambda_{G}$, resp. $\Lambda_{G}$.

The set of the $n$ by $n$ real symmetric positive semidefinite matrices will be denoted by $\mathcal{S}_{+}^{n}$, that is

$$
\mathcal{S}_{+}^{n}:=\left\{M \in \mathcal{R}^{n \times n}: M=M^{T}, u^{T} M u \geq 0\left(u \in \mathcal{R}^{n}\right)\right\} .
$$

For example, the Laplacian matrix of the graph $G$,

$$
L(G):=D_{\delta_{1}, \ldots, \delta_{n}}-A(G) \in \mathcal{S}_{+}^{n} .
$$

(Here $D_{\delta_{1}, \ldots, \delta_{n}}$ denotes the diagonal matrix with diagonal elements $\delta_{1}, \ldots, \delta_{n}$.)

It is well-known (see [9]), that the following statements are equivalent for a symmetric matrix $M=\left(m_{i j}\right) \in \mathcal{R}^{n \times n}$ : a) $M \in \mathcal{S}_{+}^{n}$; b) $\lambda_{M} \geq 0$; c) $M$ is Gram matrix, that is $m_{i j}=v_{i}^{T} v_{j}(i, j=1, \ldots, n)$ for some vectors $v_{1}, \ldots, v_{n}$. Furthermore, by Lemma 2.1 in [13], the set $\mathcal{S}_{+}^{n}$ can be described as

$$
\mathcal{S}_{+}^{n}=\left\{\left(\frac{a_{i}^{T} a_{j}}{\left(a_{i} a_{j}^{T}\right)_{11}}-1\right)_{i, j=1}^{n} \mid \begin{array}{l}
d \in \mathcal{N}, a_{i} \in \mathcal{R}^{d}(1 \leq i \leq n) \\
a_{i}^{T} a_{i}=1(1 \leq i \leq n)
\end{array}\right\} .
$$

The stability number, $\alpha(G)$, is the maximum cardinality of the (so-called stable) sets $S \subseteq V(G)$ such that $\{i, j\} \subseteq S$ implies $\{i, j\} \notin E(G)$. The chromatic number, $\chi(G)$, is the minimum number of stable sets covering the vertex set $V(G)$.

Let us define an orthonormal representation of the graph $G$ (shortly, o.r. of $G$ ) as a system of vectors $a_{1}, \ldots, a_{n} \in \mathcal{R}^{d}$ for some $d \in \mathcal{N}$, satisfying

$$
a_{i}^{T} a_{i}=1(i=1, \ldots, n), a_{i}^{T} a_{j}=0(\{i, j\} \in E(\bar{G})) .
$$


In the seminal paper [6] L. Lovász proved the following result, now popularly called sandwich theorem, see [4]:

$$
\alpha(G) \leq \vartheta(G) \leq \chi(\bar{G})
$$

where $\vartheta(G)$ is the Lovász number of the graph $G$, defined as

$$
\vartheta(G):=\inf \left\{\max _{1 \leq i \leq n} \frac{1}{\left(a_{i} a_{i}^{T}\right)_{11}}: a_{1}, \ldots, a_{n} \text { o.r. of } G\right\} .
$$

The Lovász number has several equivalent descriptions, see [6]. For example, by (1) and standard semidefinite duality theory (see e.g. [12]), it is the common optimal value of the Slater-regular primal-dual semidefinite programs

$$
(T P) \quad \min \lambda,\left\{\begin{array}{l}
x_{i i}=\lambda-1(i \in V(G)), \\
x_{i j}=-1(\{i, j\} \in E(\bar{G})), \\
X=\left(x_{i j}\right) \in \mathcal{S}_{+}^{n}, \lambda \in \mathcal{R}
\end{array}\right.
$$

and

$$
\max \operatorname{tr}(J Y),\left\{\begin{array}{l}
\operatorname{tr}(Y)=1, \\
y_{i j}=0(\{i, j\} \in E(G)), \\
Y=\left(y_{i j}\right) \in \mathcal{S}_{+}^{n} .
\end{array}\right.
$$

(Here tr stands for trace.) Reformulating the program $(T D)$, Lovász derived the following dual description of the theta number (Theorem 5 in $[6]$ ):

$$
\vartheta(G)=\max \left\{\sum_{i=1}^{n}\left(b_{i} b_{i}^{T}\right)_{11}: b_{1}, \ldots, b_{n} \text { o.r. of } \bar{G}\right\} .
$$

An important application of the theory of the theta number is described in Theorem 1 of [6], where it is proved that

$$
\Theta(G) \leq \vartheta(G)
$$

with $\Theta(G)$ denoting the Shannon capacity of the graph, that is

$$
\Theta(G):=\sup _{k \in \mathcal{N}} \sqrt[k]{\alpha\left(G^{k}\right)}
$$

(Here $G \cdot H$ denotes the strong graph product of the graphs $G, H$, the graph with vertex set

$$
V(G \cdot H):=\{(i, j): i \in V(G), j \in V(H)\}
$$

and edge set

$$
E(G \cdot H):=\left\{\begin{array}{l|l}
\left\{\left(i_{1}, j_{1}\right),\left(i_{2}, j_{2}\right)\right\} & \begin{array}{l}
i_{1}=i_{2} \text { or }\left\{i_{1}, i_{2}\right\} \in E(G) \\
j_{1}=j_{2} \text { or }\left\{j_{1}, j_{2}\right\} \in E(H)
\end{array}
\end{array}\right\} .
$$

Also, $G^{k}$ denotes the strong graph product of $k$ copies of the graph $G$.) 
The proof of (3) relies on the fact that the theta function $\vartheta($.$) is submultiplica-$ tive, that is

$$
\vartheta(G \cdot H) \leq \vartheta(G) \cdot \vartheta(H)
$$

holds for any graphs $G, H$. Another two submultiplicative bounds are described in [6], see Theorems 10 and 11; they turn out to be weaker than the theta number.

In Section 2 we will define the inverse theta number as

$$
\iota(G):=\inf \left\{\sum_{i=1}^{n} \frac{1}{\left(a_{i} a_{i}^{T}\right)_{11}}: a_{1}, \ldots, a_{n} \text { o.r. of } G\right\},
$$

and derive the inequality

$$
\alpha(G) \leq \sqrt{\iota(G)},
$$

an analogue of Lovász's sandwich theorem. In Section 3 we will prove also (as a consequence of multiplicativity properties) the stronger relation

$$
\Theta(G) \leq \sqrt{\iota(G)}
$$

It is known (see Proposition 2.2) that e.g. for the cycle graphs $C_{n}, \sqrt{\iota\left(C_{n}\right)}>$ $\vartheta\left(C_{n}\right)$ holds. Hence, the inverse theta number does not help in determining the Shannon capacity of the odd cycles $C_{7}, C_{9}, \ldots$, which is still an open problem, though, using the theta number, Lovász determined the Shannon capacity of the 5 -cycle and other graphs in [6]. However, we will see in Section 4, that orthonormal representations of the complementary graph $\bar{G}$ of high value in the dual description (5) of the inverse theta number, can be of use in a heuristic algorithm calculating large stable sets in any graph $G$.

\section{The inverse theta function}

The inverse theta number is defined via optimizing over the inverse of the theta body.

The reformulation of $\vartheta(G)$ described in (2) can be written concisely, as

$$
\vartheta(G)=\max \left\{\sum_{i=1}^{n} y_{i}: y=\left(y_{i}\right) \in T H(G)\right\},
$$

where $T H(G)$ denotes the theta body, that is the set of vectors $y=\left(y_{i}\right) \in \mathcal{R}^{n}$ such that $y_{i}=\left(b_{i} b_{i}^{T}\right)_{11}(i=1, \ldots, n)$ for some orthonormal representation $\left(b_{i}\right)$ of the complementary graph $\bar{G}$.

Convexity and compactness of the theta body follows from the fact (see Corollary 29 of [4]), that $T H(G)$ can be described equivalently as the set of vectors $y=\left(y_{i}\right) \in \mathcal{R}^{n}$ for which there exists a matrix $W=\left(w_{i j}\right) \in \mathcal{R}^{n \times n}$ satisfying both

$$
\left(\begin{array}{cc}
1 & y^{T} \\
y & W
\end{array}\right) \in \mathcal{S}_{+}^{n+1}
$$


and

$$
y_{i}=w_{i i}(i=1, \ldots, n), w_{i j}=0(\{i, j\} \in E(G)) .
$$

Analogously, let us denote by $\mathrm{TH}^{-}(G)$ the inverse theta body, that is the set of vectors $x=\left(x_{i}\right) \in \mathcal{R}^{n}$ such that $x_{i}=1 /\left(a_{i} a_{i}^{T}\right)_{11}(i=1, \ldots, n)$ for some orthonormal representation $\left(a_{i}\right)$ of the graph $G$.

From (1) it follows immediately, that $T H^{-}(G)$ can be described equivalently as the set of vectors $x=\left(x_{i}\right) \in \mathcal{R}^{n}$ such that there exists a matrix $Z=\left(z_{i j}\right) \in \mathcal{R}^{n \times n}$ satisfying

$$
z_{i i}=x_{i}-1(i=1, \ldots, n), z_{i j}=-1(\{i, j\} \in E(\bar{G})), Z \in \mathcal{S}_{+}^{n} .
$$

This fact implies the convexity of the inverse theta body, and also its monotonicity: if $\hat{x} \geq x \in T H^{-}(G)$ then $\hat{x} \in T H^{-}(G)$, too.

Let us define the inverse theta number of a graph $G$ as

$$
\iota(G):=\inf \left\{\sum_{i=1}^{n} x_{i}: x=\left(x_{i}\right) \in T H^{-}(G)\right\} .
$$

From the above considerations, and standard semidefinite duality theory (see e.g. [12]) we obtain the following statement, which implies also that the inverse theta number is efficiently computable using interior-point algorithms (see e.g. [7], [1], $[10])$.

Theorem 2.1. The inverse theta number $\iota(G)$ equals the common optimal value of the Slater-regular primal-dual semidefinite programs

$$
\begin{aligned}
& \left(T P^{-}\right) \quad \inf \operatorname{tr}(Z)+n, z_{i j}=-1(\{i, j\} \in E(\bar{G})), Z=\left(z_{i j}\right) \in \mathcal{S}_{+}^{n}, \\
& \left(T D^{-}\right) \quad \sup \operatorname{tr}(J M),\left\{\begin{array}{l}
m_{i i}=1(i=1, \ldots, n), \\
m_{i j}=0(\{i, j\} \in E(G)), \\
M=\left(m_{i j}\right) \in \mathcal{S}_{+}^{n} .
\end{array}\right.
\end{aligned}
$$

The optimal values of the programs $\left(T P^{-}\right)$and $\left(T D^{-}\right)$are attained.

Moreover, rewriting the feasible solution $M$ of the program $\left(T D^{-}\right)$as the Gram matrix $M=\left(b_{i}^{T} b_{j}\right)$ for some vectors $b_{1}, \ldots, b_{n} \in \mathcal{R}^{d}$, we obtain the following analogue of (2):

$$
\iota(G)=\max \left\{\sum_{i, j=1}^{n} b_{i}^{T} b_{j}: b_{1}, \ldots, b_{n} \text { o.r. of } \bar{G}\right\} .
$$

Similarly to $\vartheta(G)$, the number $\iota(G)$ constitutes an upper bound for the stability number $\alpha(G)$.

Theorem 2.2. For any graph $G, \alpha(G) \leq \sqrt{\iota(G)}$ holds. 
Proof. We adapt the proof of Lemma 3 in [6].

Let $S \subseteq V(G)$ be a stable set, with cardinality $\alpha(G)$. Then for any $\left(a_{i}\right)$ orthonormal representation of $G$, the vectors $a_{i}(i \in S)$ are pairwise orthogonal unit vectors. Therefore

$$
\sum_{i \in S}\left(a_{i} a_{i}^{T}\right)_{11} \leq 1
$$

which formula, by the arithmetic-harmonic mean inequality, implies that

$$
\sum_{i=1}^{n} \frac{1}{\left(a_{i} a_{i}^{T}\right)_{11}} \geq \sum_{i \in S} \frac{1}{\left(a_{i} a_{i}^{T}\right)_{11}} \geq(\alpha(G))^{2}
$$

holds. Taking infimum in $\left(a_{i}\right)$, we have the statement.

The next two propositions give in particular the exact value of $\iota(G)$ for complete multipartite graphs and for graphs with vertex-transitive automorphism group.

Proposition 2.1. For any graph $G$, the inequalities

$$
n\left(1+\frac{\mu_{\bar{G}}}{-\lambda_{\bar{G}}}\right) \leq \iota(G) \leq n\left(\mu_{\bar{G}}+1\right)
$$

hold, with equality if $G$ is a complete multipartite graph.

Proof. The inequalities are proved by the feasible solutions

$$
Z:=L(\bar{G}), M:=I+\frac{1}{-\lambda_{\bar{G}}} A(\bar{G}),
$$

which matrices have the values

$$
n\left(\mu_{\bar{G}}+1\right), n\left(1+\frac{\mu_{\bar{G}}}{-\lambda_{\bar{G}}}\right)
$$

in $\left(T P^{-}\right)$and $\left(T D^{-}\right)$, respectively.

The last assertion follows from the fact that for complete multipartite graphs $\lambda_{\bar{G}}=-1$.

The following proposition implies that for graphs with vertex-transitive automorphism group $\sqrt{\iota(G)}>\vartheta(G)$.

Proposition 2.2. For any graph $G$, the inequalities

$$
\frac{n^{2}}{\vartheta(\bar{G})} \leq \iota(G) \leq n \vartheta(G)
$$

hold, with equality if the graph $G$ has vertex-transitive automorphism group. 
Proof. First, let $\left(a_{i}\right)$ and $\left(b_{i}\right)$ be orthonormal representations of $G$ and $\bar{G}$, respectively. Then, by Lemma 4 in [6],

$$
\sum_{i=1}^{n}\left(a_{i} a_{i}^{T}\right)_{11}\left(b_{i} b_{i}^{T}\right)_{11} \leq 1
$$

holds, which formula implies, by the arithmetic-harmonic mean inequality, that

$$
\sum_{i=1}^{n} \frac{1}{\left(a_{i} a_{i}^{T}\right)_{11}\left(b_{i} b_{i}^{T}\right)_{11}} \geq n^{2} .
$$

Consequently,

$$
\max _{1 \leq i \leq n} \frac{1}{\left(b_{i} b_{i}^{T}\right)_{11}} \cdot \sum_{i=1}^{n} \frac{1}{\left(a_{i} a_{i}^{T}\right)_{11}} \geq n^{2},
$$

and taking infimum in $\left(a_{i}\right)$ and $\left(b_{i}\right)$ we have the inequality $\iota(G) \geq n^{2} / \vartheta(\bar{G})$.

On the other hand, if $M$ is feasible in $\left(T D^{-}\right)$then $Y=M / n$ is feasible in $(T D)$, which proves the inequality $\iota(G) \leq n \vartheta(G)$, too.

The last assertion follows from the fact that for graphs with vertex-transitive automorphism group, the equality $\vartheta(G) \vartheta(\bar{G})=n$ holds (see Theorem 8 in $[6]$ ).

We conclude this section with an open problem. Closedness of the convex set $T H^{-}(G)$ follows easily from the fact that $T H(\bar{G})$ is a compact set. Hence, the inverse theta body can be described as

$$
T H^{-}(G)=\bigcap_{w \geq 0}\left\{x \in \mathcal{R}^{n}: w^{T} x \geq \iota(G, w)\right\},
$$

where $\iota(G, w)$ denotes the weighted version of $\iota(G)$, that is

$$
\iota(G, w):=\inf \left\{w^{T} x: x \in T H^{-}(G)\right\}\left(w \in \mathcal{R}^{n}\right) .
$$

For special vectors $w \in \mathcal{R}^{n}$, we have seen in the proof of Proposition 2.2 that

$$
T H^{-}(G) \subseteq \bigcap_{\left(b_{i}\right)}\left\{x=\left(x_{i}\right) \in \mathcal{R}^{n}: \sum_{i=1}^{n} \frac{x_{i}}{\left(b_{i} b_{i}^{T}\right)_{11}} \geq n^{2}, x \geq 0\right\},
$$

where the $\left(b_{i}\right) \mathrm{s}$ are the orthonormal representations of the complementary graph $\bar{G}$. Does equality hold here? (For the theta body a similar linear description is known (see [4]):

$$
T H(G)=\bigcap_{\left(a_{i}\right)}\left\{y=\left(y_{i}\right) \in \mathcal{R}^{n}: \sum_{i=1}^{n}\left(a_{i} a_{i}^{T}\right)_{11} y_{i} \leq 1, y \geq 0\right\},
$$

where the $\left(a_{i}\right)$ s are the orthonormal representations of the graph $G$.) 


\section{Shannon capacity}

In this section we will prove that the inverse theta function has the same multiplicativity properties as the theta function, consequently its square root is an upper bound for the Shannon capacity of the graph.

First, we will verify the submultiplicativity of the inverse theta function, an analogue of Lemma 2 in [6].

Lemma 3.1. For any graphs $G, H, \iota(G \cdot H) \leq \iota(G) \cdot \iota(H)$.

Proof. Let $\left(a_{i}^{G}\right)$ and $\left(a_{j}^{H}\right)$ be orthonormal representations of the graphs $G$ and $H$, respectively. Then, by Lemma 1 in $[6],\left(a_{i}^{G} \otimes a_{j}^{H}\right)$ is an orthonormal representation of the graph $G \cdot H$. (Here $x \otimes y$ denotes Kronecker product of the vectors $x=\left(x_{i}\right), y$, that is the block vector $x \otimes y:=\left(x_{i} \cdot y\right)$, see [8].) Thus,

$$
\begin{aligned}
\iota(G \cdot H) & \leq \sum_{i, j} 1 /\left(\left(a_{i}^{G} \otimes a_{j}^{H}\right)\left(a_{i}^{G} \otimes a_{j}^{H}\right)^{T}\right)_{11} \\
& =\sum_{i} 1 /\left(a_{i}^{G} a_{i}^{G T}\right)_{11} \cdot \sum_{j} 1 /\left(a_{j}^{H} a_{j}^{H T}\right)_{11},
\end{aligned}
$$

and, taking infimum in $\left(a_{i}^{G}\right)$ and $\left(a_{j}^{H}\right)$, we have the statement.

Now, we will prove the skew-supermultiplicativity of the inverse theta function.

Lemma 3.2. For any graphs $G, H, \iota(\overline{\bar{G} \cdot \bar{H}}) \geq \iota(G) \cdot \iota(H)$.

Proof. Let $\left(b_{i}^{G}\right)$ and $\left(b_{j}^{H}\right)$ be orthonormal representations of the complementary graphs $\bar{G}$ and $\bar{H}$, respectively. Then, by Lemma 1 in [6], $\left(b_{i}^{G} \otimes b_{j}^{H}\right)$ is an orthonormal representation of the graph $\bar{G} \cdot \bar{H}$. Thus, by (5),

$$
\begin{aligned}
\iota(\overline{\bar{G} \cdot \bar{H}}) & \geq \sum_{i_{1}, i_{2}, j_{1}, j_{2}}\left(b_{i_{1}}^{G} \otimes b_{j_{1}}^{H}\right)^{T}\left(b_{i_{2}}^{G} \otimes b_{j_{2}}^{H}\right) \\
& =\sum_{i_{1}, i_{2}} b_{i_{1}}^{G T} b_{i_{2}}^{G} \cdot \sum_{j_{1}, j_{2}} b_{j_{1}}^{H T} b_{j_{2}}^{H},
\end{aligned}
$$

and, taking supremum in $\left(b_{i}^{G}\right)$ and $\left(b_{j}^{H}\right)$, the statement is proved.

Summarizing, we obtain the following analogue of Theorem 7 in [6].

Theorem 3.1. The inequalities in Lemmas 3.1 and 3.2 hold with equalities: for any graphs $G, H$,

a) $\iota(G \cdot H)=\iota(G) \cdot \iota(H)$;

b) $\iota(\overline{\bar{G} \cdot \bar{H}})=\iota(G) \cdot \iota(H)$. 
Proof. It is enough to notice that the graph $G \cdot H$ is a subgraph of $\overline{\bar{G} \cdot \bar{H}}$, so

$$
\iota(G \cdot H) \geq \iota(\overline{\bar{G} \cdot \bar{H}}) .
$$

Applying Lemmas 3.1 and 3.2, the proof is completed.

We remark that part of Theorem 3.1 holds also with + signs instead of $\cdot$ signs:

$$
\iota(G+H) \geq \iota(G)+\iota(H)=\iota(\overline{\bar{G}+\bar{H}}),
$$

for any graphs $G, H$. The proof of this statement is immediate from Theorem 2.1, therefore it is omitted. (For analogous results with the theta function, see [4].)

A submultiplicative upper bound for the stability number of a graph is also an upper bound for the Shannon capacity of the graph, see Theorem 1 in [6]. Consequently,

Theorem 3.2. For any graph $G, \Theta(G) \leq \sqrt{\iota(G)}$ holds.

Proof. By Theorem 2.2, for any graph $H, \alpha(H) \leq \sqrt{\iota(H)}$. Hence, from Lemma 3.1

$$
\alpha\left(G^{k}\right) \leq \sqrt{\iota\left(G^{k}\right)} \leq(\sqrt{\iota(G)})^{k}
$$

follows for $k \in \mathcal{N}$; the proof is finished.

Summarizing Theorem 1 in [6] and Theorem 3.2 we obtain

$$
\Theta(G) \leq \min \{\vartheta(G), \sqrt{\iota(G)}\} .
$$

Can $\sqrt{\iota(G)}$ be less than $\vartheta(G)$ for some graph $G$ ? Juhász's theorem (see [3]) states that $\vartheta(G)$ is typically "around" $n^{1 / 2}$ in the following sense:

Theorem 3.3. (Juhász) Let $G$ be a random graph with edge probability $p=1 / 2$. Then, with probability $1-o(1)$ for $n \rightarrow \infty$,

$$
\frac{1}{2} \sqrt{n}+O\left(n^{1 / 3} \log n\right) \leq \vartheta(G) \leq 2 \sqrt{n}+O\left(n^{1 / 3} \log n\right) .
$$

Hence, the value $\sqrt{\iota(G)}$ (which is between $n / \sqrt{\vartheta(\bar{G})}$ and $\sqrt{n \vartheta(G)}$ by Proposition 2.2) is typically "around" $n^{3 / 4}$.

Theorem 3.4. Let $G$ be a random graph with edge probability $p=1 / 2$. Then, there exist positive constants $c_{1}, c_{2}>0$ such that with probability $1-o(1)$ for $n \rightarrow \infty$,

$$
c_{1} \cdot n^{3 / 4} \leq \sqrt{\iota(G)} \leq c_{2} \cdot n^{3 / 4} .
$$

(Any $c_{1}, c_{2}>0$ such that $c_{1}^{2}<1 / 2$ and $c_{2}^{2}>2$ meet the requirements.) 
We mention two corollaries: a positive and a negative result with non-constructive proofs.

Corollary 3.1. There exist graphs $G$ such that $\sqrt{\iota(G)}<\chi(\bar{G})$.

Proof. The proof is indirect: Let us suppose that the inequality

$$
\chi(H) \leq \sqrt{\iota(\bar{H})}
$$

holds for any graph $H$.

Then, by Theorem 3.4,

$$
\alpha(H) \geq \frac{n}{\chi(H)} \geq \frac{n}{\sqrt{\iota(\bar{H})}} \geq c \cdot n^{1 / 4}
$$

would hold, with probability $1-o(1)$ as $n \rightarrow \infty$, for some appropriate constant $c>0$. On the other hand, it can easily be seen that the probability of $\alpha(H) \geq \ell$,

$$
\begin{gathered}
P(\alpha(H) \geq \ell) \leq\left(\begin{array}{c}
n \\
\ell
\end{array}\right) \cdot\left(1-\frac{1}{2}\right)^{\ell(\ell-1) / 2} \leq \\
\leq\left(n \cdot 2^{-(\ell-1) / 2}\right)^{\ell} \rightarrow 0(n \rightarrow \infty),
\end{gathered}
$$

where $\ell:=c \cdot n^{1 / 4}$. We reached contradiction with $(7)$.

Hence, there exist graphs satisfying

$$
\sqrt{\iota(\bar{H})}<\chi(H)
$$

from which, with $G=\bar{H}$, the statement follows.

From Theorems 3.3 and 3.4 immediately follows

Corollary 3.2. Under the assumptions of Theorems 3.3 and 3.4, with probability $1-o(1)$ for $n \rightarrow \infty$,

$$
\vartheta(G) \leq \sqrt{\iota(G)}
$$

Thus, the graphs $G$, with $\sqrt{\iota(G)}<\vartheta(G)$, if they exist at all, are rare. However, we will see in the following section, that the fact that $\iota(G)$ with high probability is large, can be an advance, too.

We conclude this section with an open problem: With minor modification of the proof of Theorem 2.2 it can be proved that

$$
\alpha(G)^{2} \leq \iota(G)-n+\alpha(G) .
$$

From this inequality we obtain the bound

$$
\alpha(G) \leq \frac{1}{2}(1+\sqrt{4(\iota(G)-n)+1}),
$$

which is tighter than $\alpha(G) \leq \sqrt{\iota(G)}$. It is an open problem, whether the bound in (8) is submultiplicative (and, thus, is an upper bound for the Shannon capacity $\Theta(G))$, or not. 


\section{Heuristic algorithm}

In this section we will describe a heuristic algorithm for the stable set problem.

The key observation for the algorithm is the following simple

Lemma 4.1. Let the vectors $b_{1}, \ldots, b_{n} \in \mathcal{R}^{d}$ form an orthonormal representation of the complementary graph $\bar{G}$, and let $u \in \mathcal{R}^{d}, u^{T} u=1$. Then,

$$
S:=\left\{i \in\{1, \ldots, n\}:\left(u^{T} b_{i}\right)^{2}>\frac{1}{2}\right\}
$$

is a stable set in the graph $G$.

Proof. Let us suppose indirectly that for some $i, j \in S,\{i, j\} \in E(G)$. Then, as $\left(b_{1}, \ldots, b_{n}\right)$ is an orthonormal representation of $\bar{G}$, so $b_{i}^{T} b_{j}=0$, and $\left\|b_{i}+b_{j}\right\|=\sqrt{2}$. By $i, j \in S$, we have $\left(u^{T} b_{i}\right)^{2}>1 / 2<\left(u^{T} b_{j}\right)^{2}$. Let us consider for example the case when $u^{T} b_{i}>\sqrt{2} / 2<u^{T} b_{j}$. Then,

$$
\sqrt{2}<u^{T}\left(b_{i}+b_{j}\right) \leq\|u\| \cdot\left\|b_{i}+b_{j}\right\|=\sqrt{2},
$$

which is a contradiction. The cases, when $u^{T} b_{i}<-\sqrt{2} / 2$ or $u^{T} b_{j}<-\sqrt{2} / 2$ can be dealt with similarly. This completes the proof.

Taking into account Lemma 4.1 we can search for large stable sets as follows: We compute an orthonormal representation $\left(b_{i}\right)$ of the complementary graph $\bar{G}$ and a unit vector $u$ so that $\sum_{i}\left(u^{T} b_{i}\right)^{2}$ is maximal, that is, see (2), it equals $\vartheta(G)$. (The solution of this problem is well-known, see Theorem 12 in [5].) The output stable set $S$ will be the one in (9). The algorithm derived this way is a special case of the Alon-Kahale algorithm, see Theorem 29 in [5].

To calculate with the inverse theta function $\iota(G)$ instead of the theta number $\vartheta(G)$, we take a different approach to the problem. It follows from Rayleigh's theorem and (2) that finding an orthonormal representation $\left(b_{i}\right)$ of the complementary graph $\bar{G}$ and unit vector $u$ with value $\sum\left(u^{T} b_{i}\right)^{2}=\vartheta(G)$ means solving the programs

$$
\left(P_{d}\right) \quad \sup \Lambda_{B B^{T}},\left\{\begin{array}{l}
\left(B^{T} B\right)_{i i}=1(i=1, \ldots, n) \\
\left(B^{T} B\right)_{i j}=0(\{i, j\} \in E(G))
\end{array}\right.
$$

where $B=\left(b_{1}, \ldots, b_{n}\right) \in \mathcal{R}^{d \times n}$. In other words, using the obvious equality $\Lambda_{B B^{T}}=$ $\Lambda_{B^{T} B}$ and the variable transformation $M=B^{T} B$, we have to solve the program

$$
(P) \quad \sup \Lambda_{M},\left\{\begin{array}{l}
m_{i i}=1(i=1, \ldots, n) \\
m_{i j}=0(\{i, j\} \in E(G)) \\
M=\left(m_{i j}\right) \in \mathcal{S}_{+}^{n}
\end{array}\right.
$$

This reformulation with a different proof is due to L. Lovász, who proved also the equivalence of the programs $(P)$ and $(T D)$, see [11], Theorems 11.8 and 11.3. 


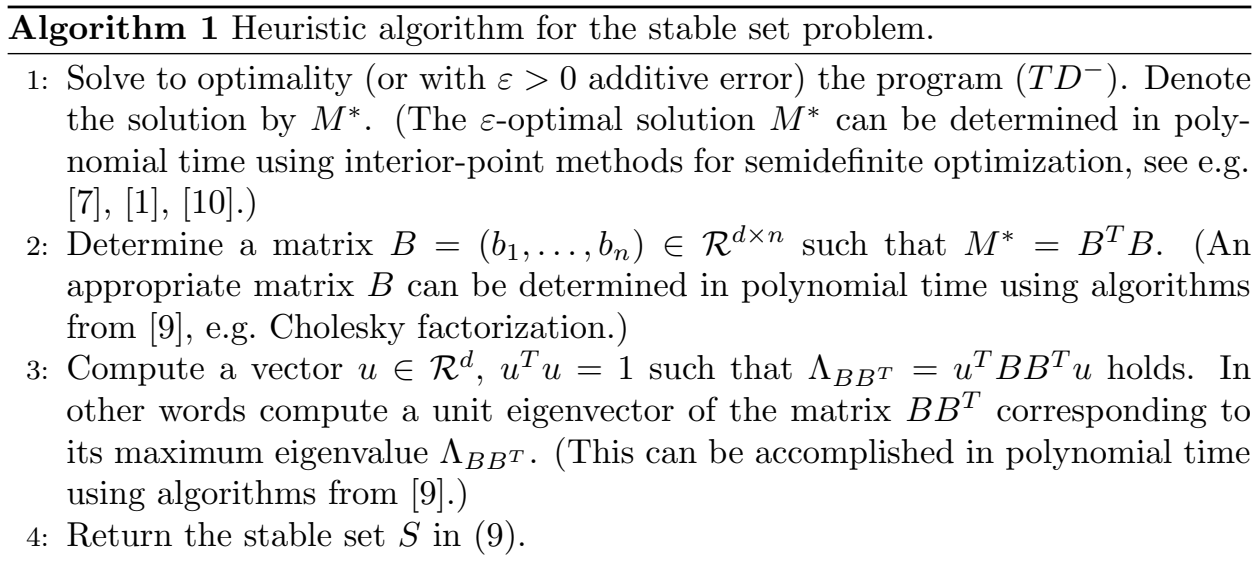

To obtain an algorithm based on the notion of the inverse theta number, instead of $(P)$ we solve the program $\left(T D^{-}\right)$for $M$, and from this matrix we compute $B, u$ and the stable set $S$. The algorithm derived this way is as follows:

We have some evidence that our algorithm finds large stable sets. Note that the following theorem implies, by Juhász's theorem, that $\sum_{i}\left(u^{T} b_{i}\right)^{2}$ is typically "around" $\sqrt{n}$ for the modified algorithm, similarly as in the case of its original version, the Alon-Kahale algorithm.

Theorem 4.1. Algorithm 1 computes an orthonormal representation $\left(b_{1}, \ldots, b_{n}\right)$ of the complementary graph $\bar{G}$, and a unit vector $u \in \mathcal{R}^{d}$ such that the inequalities

$$
\vartheta(G) \geq \sum_{i=1}^{n}\left(u^{T} b_{i}\right)^{2} \geq \frac{\iota(G)}{n} \geq \frac{n}{\vartheta(\bar{G})}
$$

hold.

Proof. The first inequality is the immediate consequence of Theorem 5 in [6]. Let us prove the second inequality. Obviously,

$$
\sum_{i=1}^{n}\left(u^{T} b_{i}\right)^{2}=\Lambda_{B B^{T}}=\Lambda_{B^{T} B}=\Lambda_{M^{*}} .
$$

On the other hand, by Rayleigh's theorem,

$$
\Lambda_{M^{*}} \geq \frac{\mathbf{1}^{T}}{\sqrt{n}} M^{*} \frac{\mathbf{1}}{\sqrt{n}}=\frac{\operatorname{tr}\left(J M^{*}\right)}{n}=\frac{\iota(G)}{n},
$$

where 1 denotes the $n$-vector with all elements equal to one. This way we have verified the inequality $\sum_{i}\left(u^{T} b_{i}\right)^{2} \geq \iota(G) / n$. Finally, the last inequality follows from Proposition 2.2. 
Note that the following corollary of Theorem 4.1 implies the relation

$$
\alpha(G) \geq \frac{2 \iota(G)}{n}-n
$$

(Similarly,

$$
\alpha(G) \geq 2 \vartheta(G)-n
$$

as the Alon-Kahale algorithm shows.)

Corollary 4.1. Algorithm 1 realizes the bound in (10): finds a stable set $S$ with cardinality $|S| \geq(2 \iota(G) / n)-n$.

Proof. The statement is an easy consequence of the inequality

$$
\sum_{i \in S}\left(u^{T} b_{i}\right)^{2}+\sum_{i \notin S}\left(u^{T} b_{i}\right)^{2} \geq \frac{\iota(G)}{n},
$$

as for $i \notin S$ we have $\left(u^{T} b_{i}\right)^{2} \leq 1 / 2$ by the definition of the stable set $S$ in (9).

Corollary 4.1 implies that $|S|>0$ if $\iota(G)>n^{2} / 2$. Thus, the output stable set $S$ is nonempty for example when $\alpha(G)>n / \sqrt{2}$.

We conclude this section with a simple example. Let us consider the graph $G=K_{s_{1}, \ldots, s_{k}}$. Then, the output matrix $M^{*}$ (the optimal solution of the program $\left.\left(T D^{-}\right)\right)$is the block-diagonal matrix made up of the matrices $J \in \mathcal{R}^{s_{1} \times s_{1}}, \ldots$, $J \in \mathcal{R}^{s_{k} \times s_{k}}$ as diagonal blocks, zero otherwise. The matrix $B \in \mathcal{R}^{k \times n}$ such that $M^{*}=B^{T} B$ is made up of the column vectors of the identity matrix $I \in \mathcal{R}^{k \times k}$ with multiplicity $s_{1}, \ldots, s_{k}$, respectively. Then, $B B^{T} \in \mathcal{R}^{k \times k}$ is the diagonal matrix with diagonal elements $s_{1}, \ldots, s_{k}$. Let us suppose that $s_{1} \geq s_{2}, \ldots, s_{k}$. Then, the vector $u \in \mathcal{R}^{k}$ equals the first column vector of the identity matrix $I \in \mathcal{R}^{k \times k}$; and $S=\left\{1, \ldots, s_{1}\right\}$ is the output stable set.

We can see that our heuristic algorithm in the case of the graph $G=K_{s_{1}, \ldots, s_{k}}$ finds a maximum stable set (and, iterating the algorithm, we obtain a minimum colouring). Generally, estimating from below the factor of the algorithm, the infimum ratio of the cardinality of the output stable set and the stability number for a graph with $n$ vertices, is an unsolved problem.

\section{Conclusion}

In this paper we studied the multiplicativity properties of the inverse theta function, and as a consequence we proved that the square root of this function is an upper bound for the Shannon capacity of the graph. Though the square root of the inverse theta number, as compared to Lovász's theta number, is typically a weak upper bound, this fact could be exploited in a heuristic algorithm for the stable set problem. 


\section{References}

[1] de Klerk, E. Interior Point Methods for Semidefinite Programming. PhD thesis, Technische Universiteit Delft, 1997.

[2] Halldórsson, M.M. Approximations of independent sets in graphs. In: Jansen, K. and Rolim, J., editors., APPROX '98, Lecture Notes in Computer Science, 1444:1-13, 1998.

[3] Juhász, F. The asymptotic behaviour of Lovász' $\vartheta$ function for random graphs. Combinatorica 2:153-155, 1982.

[4] Knuth, D. The sandwich theorem. Electronic Journal of Combinatorics, 1:148, 1994.

[5] Laurent, M. and Rendl, F. Semidefinite programming and integer programming. In: Aardal, K. et al., editors., Handbook on Discrete Optimization, Elsevier B.V., Amsterdam, pages 393-514, 2005.

[6] Lovász, L. On the Shannon capacity of a graph. IEEE Transactions on Information Theory, IT-25:1-7, 1979.

[7] Nesterov, Y. and Nemirovskii, A. Interior-Point Polynomial Methods in Convex Programming. Studies in Applied Mathematics 13, SIAM, Philadelphia, 1994.

[8] Praszolov, V.V. Lineáris Algebra. Typotex Kiadó, Budapest, 2005.

[9] Strang, G. Linear Algebra and its Applications. Academic Press, New York, 1980.

[10] Sturm, J.F. Primal-Dual Interior Point Approach to Semidefinite Programming. PhD thesis, Tinbergen Institute Research Series 156, Thesis Publishers, Amsterdam, 1997.

[11] Ujvári, M. A Szemidefinit Programozás Alkalmazásai a Kombinatorikus Optimalizálásban. Eötvös Kiadó, Budapest, 2001.

[12] Ujvári, M. A note on the graph-bisection problem. Pure Mathematics and Applications 12(1):119-130, 2002.

[13] Ujvári, M. New descriptions of the Lovász number, and the weak sandwich theorem. Acta Cybernetica 20(4):499-513, 2012. 